\title{
“Let Your Characters Tell Their Story": A Dataset for Character-Centric Narrative Understanding
}

\author{
Faeze Brahman $^{1} \quad$ Meng Huang $^{2} \quad$ Oyvind Tafjord $^{3}$ \\ Chao Zhao $^{5}$ Mrinmaya Sachan ${ }^{4} \quad$ Snigdha Chaturvedi $^{5}$ \\ ${ }^{1}$ University of California, Santa Cruz, ${ }^{2}$ University of Chicago \\ ${ }^{3}$ Allen Institute for AI, ${ }^{4} \mathrm{ETH}$ Zurich, ${ }^{5} \mathrm{UNC}$ Chapel Hill \\ fbrahman@ucsc.edu, huangme@uchicago.edu
}

\begin{abstract}
When reading a literary piece, readers often make inferences about various characters' roles, personalities, relationships, intents, actions, etc. While humans can readily draw upon their past experiences to build such a character-centric view of the narrative, understanding characters in narratives can be a challenging task for machines. To encourage research in this field of character-centric narrative understanding, we present LiSCU - a new dataset of literary pieces and their summaries paired with descriptions of characters that appear in them. We also introduce two new tasks on LiSCU: Character Identification and Character Description Generation. Our experiments with several pre-trained language models adapted for these tasks demonstrate that there is a need for better models of narrative comprehension. ${ }^{1}$
\end{abstract}

\section{Introduction}

Previous works in literary analysis have discussed that the development of the plot and the main character(s) are among the most important components that contribute to a good piece of fiction (Kennedy and Gioia, 1983; Card, 1999). In particular, char$\operatorname{acter}(s)$ are central to narratives since their motivations, traits, and actions determine the flow of the plot. Hence, understanding and critically analyzing characters is an important facet of literary scholarship.

In Computational Narratives, prior work has exploited the potential of character-centric natural language understanding (Chambers, 2013; Chaturvedi et al., 2017; Chu et al., 2018; Zhang et al., 2019). However, these works are limited to only understanding certain aspects of characters and do not do an in-depth and systematic study.

To facilitate character-centric narrative understanding, we present LiSCU - a new dataset in

\footnotetext{
${ }^{1}$ Data and code are available at: https://github.com/ fabrahman/char-centric-story
}

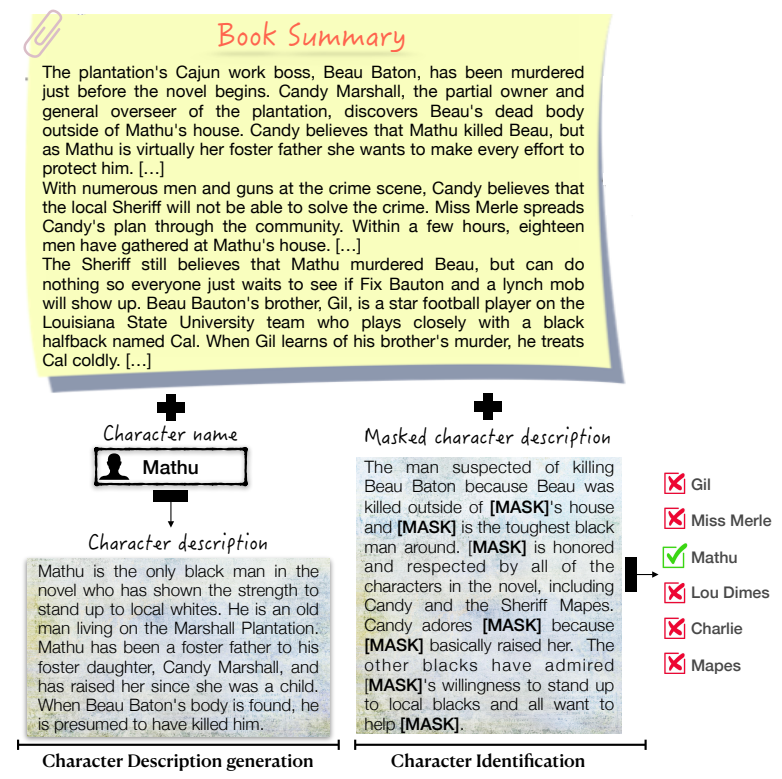

Figure 1: An illustration of the proposed dataset and the two tasks: Character Description generation and Character Identification.

English, of literary pieces and their summaries paired with descriptions of characters that appear in them. These descriptions analyze the narrative from the perspective of the character highlighting their salient attributes, their role and contribution to the development of the narrative's plot.

Using this dataset, we devise two new tasks: (1) a Character Identification task to identify the character's name from an anonymized character description given the literature summary; and (2) a Character Description Generation task to generate the description for a given character of a literature summary. Our primary task, Character Description Generation, is related, but not identical to summarization. There are two main differences. Summarization typically has a one-to-one correspondence between documents and summaries, and focuses on copying (either extractively or abstractively) important content from the documents to create the summaries. On the other hand, character 
descriptions are analysis, not merely summaries, of narratives from the character's point of view. They are created by abstracting out the low-level content of the narrative instead of simply identifying and paraphrasing important details. They describe events, roles, relationships, and salient attributes of the character that can be inferred from the narrative and might not be directly stated in the text. In particular, if the narrative describes several events where a character helps the protagonist, the character description will not simply mention all those events, but will instead describe the character as a helpful person (attribute) and a good friend of the protagonist (role). For example, in Fig. 1, "Mathu is virtually her foster father." in summary is expressed as "Candy adores Mathu because he basically raised her." in the character description. Thus, the Character Description Generation task, provides a unique opportunity for NLP systems to learn to abstract and model long-range dependency instead of simply extracting information.

Apart from this novel abstraction task, the dataset also poses another challenge for NLP systems by requiring them to process long documents. The average number of tokens in our summaries are 1022 which is beyond the comfort level of most existing systems. Understanding long narratives and modeling long contexts are new frontiers for NLP research (Roy et al., 2021; Fan et al., 2021) and LiSCU pushes us in this direction. To further facilitate research in this direction, we also release a small dataset where the goal is to read the entire literary piece and generate character descriptions.

We explore the ability of the modern neural models on both tasks. We demonstrate through experiments that although existing models can identify characters reasonably well in masked descriptions, there is still a scope for improvement considering human accuracy on this task. Also, while existing models can generate fluent and logically selfconsistent text, they are not always faithful to the literature summaries and fail to capture salient details about the characters. Our contributions are:

- A new dataset of literature summaries paired with character descriptions to enable character-centric narrative understanding.

- A comprehensive human study to assess the quality of the proposed dataset.

- Novel tasks: a classification and an abstractive generation task to better understand characters in the narrative plot.
- Experiments with several strong baselines and a thorough qualitative analysis.

\section{Background}

The field of computational narrative understanding studies how to algorithmically represent, understand, and generate stories. Early computational studies on narratives had focused on learning procedural scripts and event sequences (Schank and Abelson, 1977; Manshadi et al., 2008; Regneri et al., 2010), narrative chains or schemas (Chambers and Jurafsky, 2008, 2009), and plot units (Goyal et al., 2010; McIntyre and Lapata, 2010; Elsner, 2012).

Computational linguists have also worked on character-centric modelling of narratives (Chambers, 2013). The character-centric perspective aims to understand characters - their personas, roles, goals, relationships, emotions, etc. Previous works have proposed methods to detect characters and infer latent personas in movie plot summaries and fictional novels (Bamman et al., 2013, 2014; Vala et al., 2015; Flekova and Gurevych, 2015), model inter-character relationships (Iyyer et al., 2016; Srivastava et al., 2016; Chaturvedi et al., 2017; Kim and Klinger, 2019), and emotions (Brahman and Chaturvedi, 2020). Earlier works have also considered constructing social networks of characters (Agarwal et al., 2014) from novels (Elson et al., 2010; Elsner, 2012) and films (Krishnan and Eisenstein, 2015).

Another line of work related to ours is on summarization of novels (Mihalcea and Ceylan, 2007). This work built a dataset of novel-summary pairs and used unsupervised summarization models such as TextRank (Mihalcea and Tarau, 2004) and MEAD (Radev, 2001). Instead of summarizing full novels, Ladhak et al. (2020) proposed a contentselection approach to create a gold-standard set of extractive summaries by aligning chapter sentences with abstractive summary sentences.

In a more related work, Zhang et al. (2019) collected a dataset of fictional stories along with author-written summaries. They proposed an extractive ranking and a classification approach to select a subset of salient attributes from a list of candidate attributes (extracted from the story) that describe a character's personality. While this work presented a collection of personality-related phrases as a potential summary for the actual novel, our dataset contains literature summaries and char- 
acter descriptions, and we aim to generate natural language texts that analyze the narrative from the perspective of the characters. Such an analysis is more in-depth than a collection of phrases.

\section{The LiSCU Dataset}

We now describe our Literature Summary and Character Understanding (LiSCU) dataset. LiSCU is a dataset of literature summaries paired with descriptions of characters that appear in the summaries. Fig. 1 shows an example of our dataset.

Next, we describe the data collection pipeline for LiSCU ( $\$ 3.1)$, followed by details on the reproducibility of the data collection process $(\$ 3.2)$.

\subsection{Data Collection and Filtering}

We collected LiSCU from various online study guides such as shmoop, ${ }^{2}$ SparkNotes, ${ }_{5}^{3}$ CliffsNotes, ${ }^{4}$ and LitCharts. ${ }^{5}$ These sources contain educational material to help students study for their literature classes. These study guides include summaries of various literary pieces as well as descriptions of characters that appear in them. These literature summaries and character descriptions were written by literary experts, typically teachers, and are of high pedagogical quality.

We used Scrapy, ${ }^{6}$ a free and open-source webcrawling framework to crawl these study guides. Our initial crawl resulted in a set of 1,774 literature summaries and 25,525 character descriptions. These included all characters mentioned in the literary pieces. However, not all characters, especially those that played a minor role in the literary piece, appeared in the corresponding literature summaries. Since our task involves making inferences about characters from the literature summaries, we filtered out the characters which do not appear in the summaries or their names or the descriptions had very little overlap with the literature summaries. This is done to mitigate the reference divergence issue (Kryscinski et al., 2019; Maynez et al., 2020) and ensure that the literature summary has enough information about the character to generate the description. For this, we define the "information overlap" between two pieces of text $\mathcal{A}$ and $\mathcal{B}, I O(\mathcal{B}|| \mathcal{A})$, as the ratio of the length of the

\footnotetext{
${ }^{2}$ https://www.shmoop.com/study-guides/literature

${ }^{3} \mathrm{https}: / /$ www.sparknotes.com/lit/

${ }^{4}$ https://www.cliffsnotes.com/literature

${ }^{5}$ https://www.litcharts.com

${ }^{6}$ https://scrapy.org/
}

\begin{tabular}{l|c}
\hline \# unique books & 1,220 \\
\# literature summaries & 1,708 \\
\# characters & 9,499 \\
\# characters with accompanying full book & 2,052 \\
\# unique books with full-text & 204 \\
\hline avg. \# characters per summary & 5.56 \\
min. \# characters per summary & 1 \\
max. \# characters per summary & 38 \\
avg. summary length (in tokens) & $1,022.32$ \\
avg. \# sentences in summary & 48.82 \\
avg. character description length (in tokens) & 184.57 \\
avg. \# sentences in description & 8.56 \\
\hline \# characters in Train set & 7,600 \\
\# characters in Test set & 957 \\
\# characters in Validation set & 942 \\
\hline
\end{tabular}

Table 1: Statistics of the LiSCU dataset.

longest overlapping word sub-sequence between $\mathcal{A}$ and $\mathcal{B}$, over the length of $\mathcal{A}$. $^{7}$ Note that this information overlap measure is not symmetric and intuitively measures how much information about $\mathcal{A}$ is present in $\mathcal{B}$. We used the information overlap measure to filter our dataset as follows. If the information overlap of the literature summary with the character name, $I O$ (literature summary $\|$ character name), is less than 0.6 , then we consider that the character is not prominently mentioned in the literature summary and we remove that character from our dataset. Similarly, if the information overlap between the character description and the literature summary, $I O$ (literature summary $\|$ character description), is less than 0.2 , then we consider the character description generation less feasible and we remove that data point from our dataset. ${ }^{8}$

However, during these filtering steps, we did not want to remove the most important characters of the narrative. The online study guides list characters in decreasing order of their importance in the literary piece. For example, narrators, protagonists, antagonists, etc., are always described first. Leveraging this ordering, we always retained the top 3 characters of the literary piece in our dataset.

After the filtering process, our final dataset consists of 1, 708 literature summaries and 9, 499 character descriptions in total. This set was split into train $(80 \%)$, test $(10 \%)$, and validation $(10 \%)$ sets.

\footnotetext{
${ }^{7}$ Technically this is the same as Rouge-L precision

${ }^{8}$ These thresholds were chosen by experimenting with different values and manually analyzing the quality of (a subset of) the data.
} 
The data splits were created to avoid any dataleakages - each literary piece and all of its character descriptions were consistently part of only one of the train, test and validation sets. Table 1 shows the statistics of the final dataset. The dataset also contains the full-text of the books for 2,052 of the character descriptions.

\subsection{Dataset Reproducibility}

LiSCU is drawn from various study guides on the web. While we do not have the rights to directly redistribute this dataset, to allow other researchers to replicate the LiSCU dataset and compare to our work, we provide a simple script that will allow others to recreate LiSCU from a particular timestamped version of these study guides on Wayback Machine, a time-stamped digital archive of the web. Our script ensures that others will be able to recreate the same train, test and validation splits.

\section{LiSCU Task Definitions}

We introduce two new tasks on the LiSCU dataset:

- Character Identification

- Character Description Generation

\subsection{Character Identification}

The Character Identification task requires models to identify the character in an anonymized character description. Given a summary $S$, a candidate list of characters that appear in the literature summary $C=\left\{c_{1}, c_{2}, \ldots, c_{k}\right\}$, and an anonymized character description $D_{\text {masked }}^{c *}$, the goal in this task is to identify the name of the character $c^{*}$ described in the anonymized character description. We anonymize character descriptions by masking out all mentions of the character $c^{*}$ in the original description $D^{c *}$.

\subsection{Character Description Generation}

The character description generation task tests the ability of NLP models to critically analyze the narrative from the perspective of characters and generate coherent and insightful character descriptions. Formally, given a literature summary, $S$, and a character name, $c$, the goal in this task is to generate the character's description, $D^{c}$. Generating the character description necessitates understanding and analyzing every salient information about the character in the literature summary.

\subsection{Human Assessment of LiSCU}

In order to verify the tractability of these two tasks as well as assessing the quality of the collected

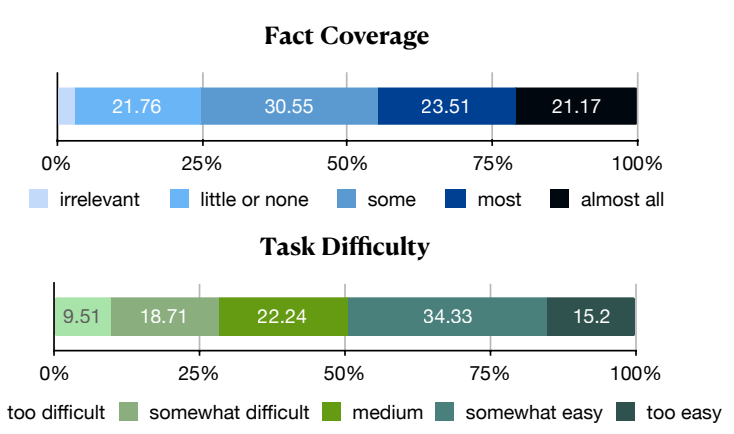

Figure 2: Human assessment of the feasibility of the character description generation task.

LiSCU dataset, we conducted a set of human evaluations on Amazon Mechanical Turk. We run our human assessment on the full test set of LiSCU.

Assessing the Character Identification task: In the first human assessment, we showed annotators the literature summaries, anonymized character descriptions, and a list of character names (plus one randomly sampled character from the literary piece). The descriptions were anonymized by replacing all mentions of the corresponding character names with blanks. ${ }^{9}$ For each anonymized character description, we asked 3 judges to identify which character it is describing by choosing from the list of choices. The judges also had the option of saying that they are unable to identify the character given the literature summary and the anonymized character description.

Assessing the Character Description Generation task: In the second human assessment, the judges are shown the same summary along with the original de-anonymized character descriptions. For each character description, 3 judges were asked to evaluate the quality of the description by answering the following two questions:

1. Fact coverage: Specify how much of the information about the specific character in the corresponding "character description" is present in the summary (either explicitly or implicitly). Answer choices included: a) almost all of the information, b) most of the information, c) some of the information, d) little or none of the information, and e) character does not appear in the summary at all.

2. Task difficulty: Given the summary, how easy is it to write the character description on a Likert scale of 0-4 ( 0 being too difficult, 4 being too

\footnotetext{
${ }^{9}$ We identified mentions of a character in the summary by using a coreference system (Joshi et al., 2019b,a) as well as by matching the first name or the full name of the character.
} 


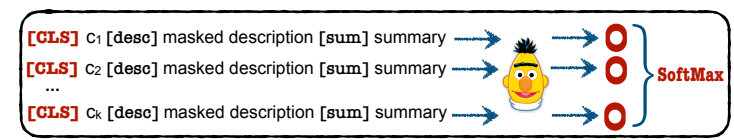

(1) Multiple-choice: fine-tuning RoBERTa/ALBERT

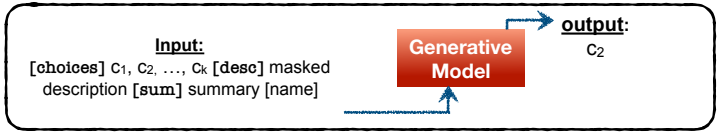

(2) Generative: fine-tuning T5/BART/Longformer in text-to-text format.

Figure 3: Approaches for Character Identification.

easy)? If in the previous question the judges found that some of the information in the character description was not present in the summary, they are asked to disregard that while answering this question. In other words, they only need to consider the information in the character description which is explicitly or implicitly mentioned in the summary.

We recruited 200 crowd-workers who were located in the US, UK, or CA, and had a $98 \%$ approval rate for at least 5,000 previous annotations. We collected each annotation from 3 workers and use majority vote in our assessments. In the Appendix A, we describe several steps we took to alleviate limitations of using crowd-sourcing and ensure high quality annotations. Screenshots of our AMT experiments are provided in the Appendix.

For the first assessment on identifying characters, the human accuracy was $91.80 \%$ (Fleiss' Kappa (Landis and Koch, 1977) $\kappa=0.79$ ), indicating the feasibility of the task.

For the second assessment of fact coverage and task difficulty, we summarize the result in Fig. 2. The top chart ('Fact Coverage') shows that around $75 \%$ of the of the literature summaries contain reasonable amount of information about the character represented in the corresponding character description. The bottom chart ('Task Difficulty') shows that more than $90 \%$ of the times, the human judges considered the task of writing the character descriptions from the literature summaries not too difficult. $^{10}$

These results verify the feasibility of understanding and drawing reasonable inferences about characters in the literature summaries from the LiSCU

\footnotetext{
${ }^{10}$ There is a natural label bias in the annotations: most of the responses fell into few categories. In this case, standard interannotator agreement statistics are not reliable (the well-known paradoxes of kappa (Feinstein and Cicchetti, 1990)). Thus, we simply report a pairwise agreement (i.e., how often do two judges agree on the answer for the same question) of 0.71 and 0.64 for 'fact coverage' and 'task difficulty', respectively.
}

\begin{tabular}{lcc}
\hline Model & Description Setup & Accuracy (\%) \\
\hline Random Guess & - & 18.70 \\
\hline RoBERTa-Large (Liu et al., 2019) & partial & 77.84 \\
ALBERT-XXL (Lan et al., 2020) & partial & $\mathbf{8 3 . 3 3}$ \\
\hline T5-11B (Raffel et al., 2020a) & partial & $\underline{80.16}$ \\
BART-Large (Lewis et al., 2019) & partial & 74.89 \\
Longformer (Beltagy et al., 2020) & partial & 71.10 \\
BART-Large (Lewis et al., 2019) & full & 78.58 \\
Longformer (Beltagy et al., 2020) & full & 74.78 \\
\hline Human Performance & - & 91.80 \\
\hline
\end{tabular}

Table 2: Accuracy for the Character Identification. The 'partial' description setup used a truncated description ( 50 words) to allow including more of the summary.

dataset. Next, we describe models and establish baseline performances on the two proposed tasks.

\section{Character Identification}

We present two approaches to address this task: (1) solving it as a multiple-choice classification problem, and (2) using a generative classifier that generates, instead of identifying, the character name, as shown in Fig. 3.

In the multiple-choice approach, we use the standard setup introduced in BERT (Devlin et al., 2019) where the text from $c_{i}, D_{\text {masked }}^{c *}$ and $S$ (with custom prefix tokens) are concatenated as input, and the [CLS] token is projected to a final logit. We apply a Softmax function to the logits to obtain the scores for each $c_{i}$. For training practicalities, we limit the number of choices to 4 during training (using the earliest window of choices which include the correct one). During inference, we can generate the logits for all the answer choices since they are independent before the final Softmax.

To establish a baseline performance, we experiment with finetuning RoBERTa (Liu et al., 2019), and ALBERT (Lan et al., 2020) which have been shown to perform well in several classification tasks. However, both these models cannot process inputs longer than 512 tokens and the concatenated inputs are generally much longer. So we also tried Longformer (Beltagy et al., 2020), a BERT-like model with an attention mechanism designed to scale linearly with sequence length, thus allowing the model to encode longer documents. However, despite trying various hyperparameters, Longformer was not able to match the scores in our experiments.

Our second approach, a generative classifier, is 
inspired by Raffel et al. (2020b) who studied transfer learning by converting NLP problems into a text-to-text format. The generative classifier addresses the character identification problem by directly generating the character name $\hat{c}$, given all character names (answer choices), the masked character description, and the summary (see Fig. 3). During inference, we compute the model's probability of each of the answer choices, and output the one with the highest probability.

We use this procedure to train several strong baselines built on top of the following pre-trained transformer-based models: BART (Lewis et al., 2019), T5 (Raffel et al., 2020b), and Longformer (Beltagy et al., 2020).

Implementation Details. The RoBERTa and ALBERT multiple-choice classifiers were trained for 6 epochs, initial learning rate 1e-5 (ADAM optimizer), batch size 16 . The generative classifier using BART was trained for 5 epochs, initial learning rate $5 \mathrm{e}-6$, batch size 8 . We used the Transformer package (Wolf et al., 2019) for training. The T5 model was trained for 12 epochs on a TPU using the default parameters from the T5 repository (learning rate 1e-3 with AdaFactor, batch size 8). ${ }^{11}$ We truncate the summaries (and descriptions) to satisfy model-specific maximum input length.

Results. Table 2 shows the accuracies of different baselines. The highest accuracy is achieved by ALBERT-XXL (83.33\%) followed by T5-11B (80.16\%). Although both ALBERT and T5 were given partial character descriptions, their specific pre-training loss and larger number of parameters (for T5-11B) lead to superior performance over other baselines. We observe that there is still a significant difference between the human performance $(91.80 \%)$ and the best model performance $(83.33 \%)$ on the character identification task, warranting future work on this direction.

\section{Character Description Generation}

We present several strong baselines for generating character descriptions by fine-tuning pre-trained transformer-based language models (LM) (Vaswani et al., 2017). We study two types of models: (1) a standard left-to-right LM, namely GPT2-L (Radford et al., 2019) which is trained with LM objective to predict the next word; and (2) two encoder-

\footnotetext{
${ }^{11}$ https://github.com/google-research/ text-to-text-transfer-transformer
}

\begin{tabular}{lccccc}
\hline Model & BLEU & ROUGE-1 & ROUGE-2 & ROUGE-L & BERT-F1 \\
\hline \multicolumn{5}{c}{ Length Truncated Input } \\
GPT2-L & 0.67 & 19.25 & 3.50 & 17.51 & 77.71 \\
BART-L & $\mathbf{1 . 3 8}$ & $\mathbf{2 4 . 9 3}$ & $\mathbf{5 . 4 2}$ & $\mathbf{2 1 . 9 9}$ & 84.54 \\
Longformer & 1.05 & 21.47 & 4.66 & 19.37 & 84.64 \\
\hline \multicolumn{5}{c}{ Coref Truncated Input } \\
GPT2-L & 0.58 & 18.69 & 3.15 & 16.91 & 78.46 \\
BART-L & 0.96 & 21.33 & 4.66 & 19.04 & 84.26 \\
Longformer & 0.98 & 21.18 & 4.40 & 19.13 & 84.59 \\
\hline \multicolumn{5}{c}{ Full Length Input } \\
Longformer & 1.14 & 21.79 & 4.88 & 19.60 & $\mathbf{8 4 . 7 2}$ \\
\hline
\end{tabular}

Table 3: Automatic evaluation results for Character Description Generation. BART-L achieved the best BLEU and ROUGE scores while Longformer performed best on BERTScore.

decoder models, namely BART ${ }^{12}$ (Lewis et al., 2019) and Longformer (Beltagy et al., 2020) ${ }^{13}$ which initialize the state of the Transformer by reading the input, and learn to generate the output.

One of the challenges of the proposed task is the length of the summaries, which might exceed the maximum allowable length for most existing pre-trained models. To overcome this, we either: (1) simply truncate the literature summary at the end, or (2) only keep sentences from the literature summary that have a mention of the character of interest. For the latter, we use a coreference resolution model, SpanBERT (Joshi et al., 2019b,a), to identify character mentions within a summary. This results in a modified dataset of character-specific literature summaries paired with character descriptions. In addition to these two approaches, we also fine-tune Longformer (Beltagy et al., 2020) with original full-length literature summary. Longformer leverages an efficient encoding mechanism to avoid the quadratic memory growth and has been previously explored for NLU tasks (encoder-only). We integrate this approach into the pre-trained encoder-decoder BART model to encode inputs longer than its maximum token limit. All the models take [name] $c$ [sum] $S$ [desc] as input and generate the character description $D^{c}$ as output.

Experiment with Full Literary Pieces. We also run an experiment on a subset of our data with accompanying full-text of the literary pieces.

\footnotetext{
${ }^{12}$ We use the bart-large-xsum as initial weights as our task can benefit from the summarization capability.

${ }^{13} \mathrm{https}$ ///github.com/allenai/longformer. We initialize parameters of Longformer with the same pre-trained BART.
} 


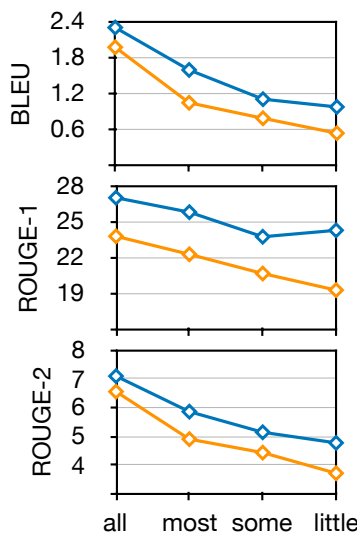

$\diamond$ BART-L (length-truncated) BART-L (coref-truncated)

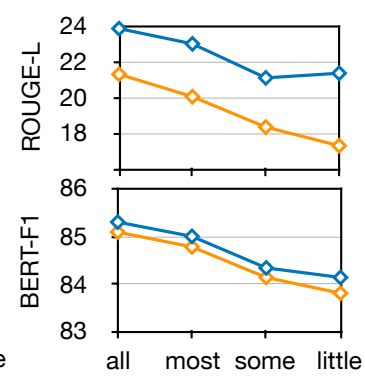

Figure 4: Breakdown results for BART-L on subsets with annotated fact coverage as all/most/some/little. Results for other baselines are provided in Appendix.

Since it is infeasible to use the full texts as input given the memory constraints of current models, we coarsely select spans of the full-text beginning 50 tokens before, and 50 tokens after the occurrence of character's name. We use a Longformer model where the input is simply the concatenation of the selected spans. Due to the small size of the this subset, we perform a 5 -fold cross validation starting from a pre-trained model fine-tuned on summary-description pairs. ${ }^{14}$

Implementation Details. We use the Transformer library (Wolf et al., 2019). Each baseline was trained for 5 epochs with effective batch size of 8 , and initial learning rate of $5 \mathrm{e}-6$. We use the maximum input length of 1024 for GPT2, and 2048 for BART $^{15}$ and the variant of Longformer with truncated input. For experiment with original books, we use 16,384 which is the maximum allowable input length for Longformer. During inference, we use beam search decoding with 5 beams.

\subsection{Automatic Evaluation}

Following previous works, we use several standard, widely used automatic evaluation metrics. We use BLEU-4 (Papineni et al., 2002) that measures overlap of $n$-gram up to $n=4$, ROUGE- $n(n=1,2)$, and ROUGE-L F-1 scores (Lin, 2004) ${ }^{16}$. However, recent works (Novikova et al., 2017; Wang et al., 2018) have raised concerns on the usage of these metrics as they fail to capture paraphrases

\footnotetext{
${ }^{14}$ Pre-training data do not contain instances of this subset.

${ }^{15}$ BART originally accepts inputs of maximum 1024 BPEtokens. We extend this to 2048 by adjusting its positional embeddings.

${ }^{16}$ Note that we did not include perplexity score as it is not comparable across LM-based and encoder-decoder models.
}

\begin{tabular}{lccc}
\hline Model & BLEU & R-1/ R-2 /R-L & BERT-F1 \\
\hline Longformer (w/ Books) & 0.73 & $17.61 / 3.60 / 16.15$ & 84.33 \\
Longformer (w/ Summaries) & $\mathbf{1 . 0 0}$ & $\mathbf{1 9 . 4 6 / 4 . 3 3 / 1 7 . 7 4}$ & $\mathbf{8 4 . 7 7}$ \\
\hline
\end{tabular}

Table 4: Automatic evaluation results for models using full-text of books vs. literature summaries.

and conceptual information. To overcome these issues, we additionally include a model-based metric, BERTScore (Zhang et al., 2020), which measures the cosine similarity between contextualized embeddings of the gold and generated outputs. ${ }^{17}$

The result of the automatic evaluation is presented in Table 3. According to the table, BART-L consistently achieves the best performance across BLEU and ROUGE scores. However, Longformer achieves a slightly better BERTScore. Both BART and Longformer outperform GPT2 in general. This can be in part because BART and Longformer can handle longer context, and are initially pre-trained on a combination of books and Wikipedia data and further fine-tuned on summarization tasks, while GPT2 is pre-trained on WebText only. ${ }^{18}$

Models perform relatively better in the length truncation setups than in the coreference truncation. We posit that this is because a lot of the key points about major characters are likely to appear earlier in the book summary (favoring length truncation). Also, there might be errors introduced by the coreference resolution model itself.

In order to have a better insight into the models' performance with respect to varying level of task feasibility, in Fig. 4, we additionally report the breakdown of the results for BART-L on separate subsets with "almost all", "most", "some", "little or none" of the information about the character (refer to Fact Coverage in §4.3). As expected, we observe a consistent decline in the performance with lower amount of fact coverage. Results for other baselines are reported in Table 8 of the Appendix.

In Table 4, we compare the models when using selected spans from the original literary piece as the input vs. literature summaries as the input. We observe a decline in performance when we used the full text. This reveals that even though the literary pieces contain all the character information, this information is scattered which makes it harder for

\footnotetext{
${ }^{17}$ We use the code at https://github.com/Tiiiger/bert_score

${ }^{18}$ While these models could have had access to the original book text, they do not have access to the character descriptions (our outputs) during pre-training. So, this information should not principally change any of our empirical conclusions.
} 


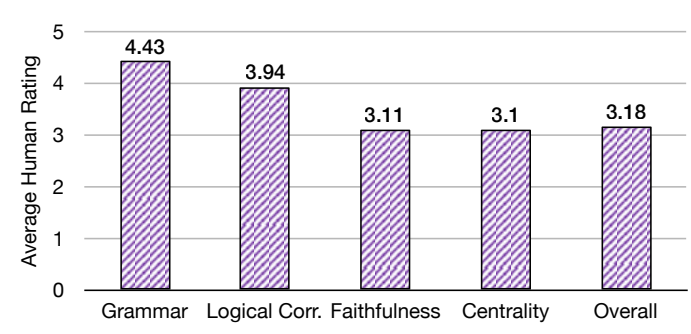

Figure 5: Human evaluation of generated character descriptions. While the descriptions are grammatically correct and logically coherent, they often misrepresent or miss important details about the character.

the model to identify important facts about the character. Using full texts also requires encoders which are better at understanding dialog, first-person narratives and different writing styles of the authors. We invite the community to consider this challenging but important problem.

\subsection{Human Evaluation}

To better evaluate the quality of the generated character descriptions, we conduct a human evaluation on 100 test pairs of literature summaries and character descriptions generated by the BART-L model on Amazon Mechanical Turk. ${ }^{19}$ Given a literature summary and multiple generated character descriptions (shown one by one), the workers were asked to rate each generated description on a Likert scale of $1-5$ ( 1 being the worst, and 5 being the best) according to the following criteria: (1) Grammatical correctness to indicate if the generated description is grammatically correct, (2) Logical correctness to indicate whether the generated description is logically meaningful and coherent, (3) Faithfulness of the generated description with respect to the given summary (a faithful character description will not mention facts which are irrelevant to the character and/or not stated in the summary), (4) Centrality to evaluate whether the description captures important details and key facts about the character, and finally (5) the Overall score considering all the four criteria listed above. We provide a screenshot of the experiment in Fig. 7 of the Appendix.

Fig. 5 presents the results of this human evaluation. We observe that the generated descriptions show a reasonable level of grammatical (4.43) and logical correctness (3.94). However, they lack behind when it comes to faithfulness (3.11) and centrality (3.10). We also report the distribution of

\footnotetext{
${ }^{19}$ Here we are evaluating 4 character descriptions per summary, for the total of 25 literature summaries.
}

\begin{tabular}{lccccc}
\hline Aspects & (1) & (2) & (3) & (4) & (5) \\
\hline Grammar & 0.00 & 3.67 & 8.67 & 28.67 & 59.00 \\
Logical Corr. & 1.67 & 9.00 & 19.00 & 33.67 & 36.67 \\
Faithfulness & 12.67 & 23.67 & 21.00 & 24.67 & 18.00 \\
Centrality & 15.33 & 17.00 & 27.00 & 23.67 & 17.00 \\
Overall & 11.00 & 19.33 & 26.67 & 26.67 & 16.33 \\
\hline
\end{tabular}

Table 5: Percentage of different ratings from human evaluation of generated descriptions ( $1=$ worst, $5=$ best).

\begin{tabular}{lc}
\hline Error Type & Percentage \\
\hline Events & 46.00 \\
Role & 24.33 \\
Relationships & 25.00 \\
Personal characteristics & 12.33 \\
Behavioral characteristics & 22.33 \\
No major error & 27.67 \\
\hline
\end{tabular}

Table 6: Error Analysis: proportion of generated descriptions with different error types.

ratings in Table 5. These results indicate that solving this task requires designing better models of character-centric analysis of narrative.

\subsection{Qualitative Analysis}

Here, we do a qualitative analysis for the Character Description Generation task. In our human evaluation of the generated character descriptions (\$6.2), we additionally provided a questionnaire to collect in-depth feedback from crowd-workers on the type of errors the BART-L model made when generating character descriptions from the given literature summaries. The questionnaire asked "What details about the character does the given character description miss or describe inaccurately. Note that the description is supposed to describe only the important details and not necessarily all of them." The workers were asked to select all the applicable choices among the following error types:

1. Events: The character description misses or misrepresents some main event(s) that the character is involved in.

2. Role: The character's role in the narrative (e.g., protagonist, antagonist, etc.) is important but is not included or misrepresented in the character description.

3. Relationships: The character's relationship with other characters is important (e.g., the protagonist's wife) but is not included or misrepresented in the character description.

4. Personal characteristics: The character's 


\begin{tabular}{|c|c|}
\hline Generated Character Descriptions & Comments \\
\hline $\begin{array}{l}\text { Book title: The Three Sisters } \\
\text { Character name: Vershinin } \\
\text { Generated Character Description: Vershinin is one of the most interesting characters } \\
\text { in Three Sisters. He's a young officer in the Russian army, and he's got a lot of time on } \\
\text { his hands. But that doesn't stop him from having an affair with Irina, the youngest sister, } \\
\text { who falls in love with him because she's attracted to his philosophical ideas about the } \\
\text { meaning of life. }\end{array}$ & $\begin{array}{l}\text { According to the sum- } \\
\text { mary "Masha", the mid- } \\
\text { dle sister, is the one who } \\
\text { has an affair with the } \\
\text { officer Vershinin, and } \\
\text { not the youngest sister, } \\
\text { Irina. }\end{array}$ \\
\hline $\begin{array}{l}\text { Book title: The Sun Also Rises } \\
\text { Character name: Pedro Romero } \\
\text { Generated Character Description: Pedro Romero is a young Spanish bull-fighter who } \\
\text { is in love with Lady Brett Ashley, a beautiful British divorcee. He's a talented and } \\
\text { talented young man, and, despite the fact that he is only twenty-five years old, he has the } \\
\text { potential to be one of the best bullfighters in Pamplona. Jake feels terrible for introducing } \\
\text { him to Brett, fearing that it has corrupted him. }\end{array}$ & $\begin{array}{l}\text { The description cap- } \\
\text { tures most of the im- } \\
\text { portant details about the } \\
\text { character. }\end{array}$ \\
\hline
\end{tabular}

Table 7: Examples of generated descriptions. Words in red correspond to hallucinated or missing content, and words in green correspond to faithful information. The input literature summaries are provided in the Appendix.

personal characteristics (e.g., age, ethnicity, personality, etc.) are important for the narrative but are not included or misrepresented in the character description.

5. Behavioral characteristics: The character's motivation, desires, and behavior are important but are not included or misrepresented in the character description.

6. No major error: None of the above. The character description captures most of the important details about the character.

We also provided an optional text box for them to type in other details that are missing or misrepresented but not listed above.

The result of this analysis is shown in Table 6 . We can see that the generated descriptions make fewer mistakes in capturing personality-related attributes (12.33\%) and more mistakes in representing important events involving the characters (46\%). They also sometimes omit or misrepresent roles $(24 \%)$, relationships ( $25 \%$ ), and behavioral characteristics $(22 \%)$ of the characters. This indicates factors that future systems should consider improving upon when addressing this task.

We provide qualitative examples of the generated character descriptions along with the errors they made (as pointed out by the turkers) in Table 7 . More examples with input literature summaries are provided in Tables 9 to 12 of the Appendix.

\section{Conclusion}

Understanding and critically analyzing fictional characters is an important element of understand- ing a literary piece. Human readers build a mental model of characters, understand what they look like, their role in the literary piece, and assess their psychology, motivations, and consequences of their behavior. However, building such a deep understanding of fictional characters in narratives is hard for machine reading systems. To encourage progress in character-centric understanding of narratives, we present LiSCU, a dataset of literature summaries paired with descriptions of characters that appear in them. We use LiSCU to propose two tasks that explore the ability of the modern neural models to understand the narrative from the perspective of characters. Performing human assessments on the model outputs show that there is still a lot of room for improvement on these tasks.

\section{Acknowledgments}

This work was supported in part by ETH Grant (ETH-19 21-1) and NSF grant IIS2047232. We would also like to thank Jena D. Hwang for helping with designing the AMT task.

\section{Broader Impacts and Ethics Statement}

Bias in Narrative Texts: LiSCU is based on novels which often reflect societal norms and biases of their times. Such a dataset can be used to understand societal bias as well as design Natural Language Understanding models that can be more aware of and possibly even avoid such biases. With this motivation, we analyzed the issue of gender bias in LiSCU. 
First, we inferred the gender of the characters in our dataset using the pronouns used to refer to them. We could not infer the gender of some of the characters because of errors in the coreference system or lack of enough mentions, and we filtered them out for this analysis. We found that there are significantly more male characters than female characters in our dataset. Specifically, $66 \%$ of the characters are male. This suggests that systems that do not account for this bias might end up having more training data (and hence yield better performance) on descriptions of male characters than of female characters.

Second, we also investigated the scope of gender bias in the summaries. We computed the average number of mentions of male and female characters (in the summaries). We found that on average male and female characters are mentioned 32.1 and 31.7 times, respectively. This indicates that even though there are fewer female characters in the literary pieces of our dataset, the ones that are present play a significant role in the development of the narrative. Possibly because of their importance in the narrative, they are mentioned as many times as male characters in the summary (which describes the main developments and not all details from the literary piece).

Third, we investigated if the literary experts who composed the descriptions were biased in their analysis. For this, we compute the length of character descriptions of various characters. We found that there is no significant difference between male and female characters in this aspect. Specifically, the average number of tokens in the description of a male character was 203 , and that of a female character was 200. Also, the average number of sentences in the description of a male character was 9.4 and that of a female character was 9.3. This also aligns with our observation in the previous experiment where we found that female characters, though fewer, play important roles in the narrative, and so their descriptions are not any shorter than descriptions of male characters. Overall, this analysis suggests that descriptions are not biased in their treatment of male and female characters.

In any language generation setting, such as ours, there is the possibility of (potentially harmful) social biases that can be introduced in the training data. As we did not specifically control or regularize our model to remove the possibility of such biases, we would urge downstream users to un- dertake the necessary quality-assurance testing to evaluate the extent to which such biases might be present and impacting their trained system and to make modifications to their model and procedures accordingly.

Human participation in our study : We conducted 2 human evaluations on Amazon Mechanical Turk. To ensure the annotators were fairly compensated, we did several rounds of test runs and estimated the average time to finish one HIT. Workers were paid $\$ 12 / \mathrm{hr}$ based on the HIT timings. We did not ask any personal, sensitive or identifying information from the annotators.

\section{References}

Apoorv Agarwal, Sriramkumar Balasubramanian, Anup Kotalwar, Jiehan Zheng, and Owen Rambow. 2014. Frame semantic tree kernels for social network extraction from text. In Proceedings of the 14th Conference of the European Chapter of the Association for Computational Linguistics, pages 211219, Gothenburg, Sweden. Association for Computational Linguistics.

David Bamman, Brendan O'Connor, and Noah A. Smith. 2013. Learning latent personas of film characters. In Proceedings of the 51st Annual Meeting of the Association for Computational Linguistics (Volume 1: Long Papers), pages 352-361, Sofia, Bulgaria. Association for Computational Linguistics.

David Bamman, Ted Underwood, and Noah A. Smith. 2014. A Bayesian mixed effects model of literary character. In Proceedings of the 52nd Annual Meeting of the Association for Computational Linguistics (Volume 1: Long Papers), pages 370-379, Baltimore, Maryland. Association for Computational Linguistics.

Iz Beltagy, Matthew E. Peters, and Arman Cohan. 2020. Longformer: The long-document transformer. ArXiv, abs/2004.05150.

Faeze Brahman and Snigdha Chaturvedi. 2020. Modeling protagonist emotions for emotion-aware storytelling. In Proceedings of the 2020 Conference on Empirical Methods in Natural Language Processing (EMNLP), pages 5277-5294, Online. Association for Computational Linguistics.

Orson Scott Card. 1999. Elements of Fiction Writing Characters \& Viewpoint. Writer's Digest Books.

Nathanael Chambers. 2013. Event schema induction with a probabilistic entity-driven model. In Proceedings of the 2013 Conference on Empirical Methods in Natural Language Processing, pages 1797-1807, Seattle, Washington, USA. Association for Computational Linguistics. 
Nathanael Chambers and Dan Jurafsky. 2008. Unsupervised learning of narrative event chains. In Proceedings of ACL-08: HLT, pages 789-797, Columbus, Ohio. Association for Computational Linguistics.

Nathanael Chambers and Dan Jurafsky. 2009. Unsupervised learning of narrative schemas and their participants. In Proceedings of the Joint Conference of the 47th Annual Meeting of the ACL and the 4th International Joint Conference on Natural Language Processing of the AFNLP, pages 602-610, Suntec, Singapore. Association for Computational Linguistics.

Snigdha Chaturvedi, Mohit Iyyer, and Hal Daumé III 2017. Unsupervised learning of evolving relationships between literary characters. In Proceedings of the Thirty-First AAAI Conference on Artificial Intelligence, , pages 3159-3165.

Eric Chu, Prashanth Vijayaraghavan, and Deb Roy. 2018. Learning personas from dialogue with attentive memory networks. In Proceedings of the 2018 Conference on Empirical Methods in Natural Language Processing, pages 2638-2646, Brussels, Belgium. Association for Computational Linguistics.

Jacob Devlin, Ming-Wei Chang, Kenton Lee, and Kristina Toutanova. 2019. BERT: Pre-training of deep bidirectional transformers for language understanding. In $N A A C L$, pages 4171-4186.

Micha Elsner. 2012. Character-based kernels for novelistic plot structure. In Proceedings of the 13th Conference of the European Chapter of the Association for Computational Linguistics, pages 634-644, Avignon, France. Association for Computational Linguistics.

David K. Elson, Nicholas Dames, and Kathleen R. McKeown. 2010. Extracting social networks from literary fiction. In ACL 2010, Proceedings of the 48th Annual Meeting of the Association for Computational Linguistics, July 11-16, 2010, Uppsala, Sweden, pages 138-147. The Association for Computer Linguistics.

Angela Fan, Thibaut Lavril, Edouard Grave, Armand Joulin, and Sainbayar Sukhbaatar. 2021. Addressing some limitations of transformers with feedback memory.

Alvan R Feinstein and Domenic V Cicchetti. 1990 High agreement but low kappa: I. the problems of two paradoxes. Journal of clinical epidemiology, 43(6):543-549.

Lucie Flekova and Iryna Gurevych. 2015. Personality profiling of fictional characters using sense-level links between lexical resources. In Proceedings of the 2015 Conference on Empirical Methods in Natural Language Processing, pages 1805-1816.

Amit Goyal, Ellen Riloff, and Hal Daumé III. 2010 Automatically producing plot unit representations for narrative text. In Proceedings of the 2010 Conference on Empirical Methods in Natural Language Processing, pages 77-86, Cambridge, MA. Association for Computational Linguistics.

Mohit Iyyer, Anupam Guha, Snigdha Chaturvedi, Jordan Boyd-Graber, and Hal Daumé III. 2016. Feuding families and former friends: Unsupervised learning for dynamic fictional relationships. In Proceedings of the 2016 Conference of the North American Chapter of the Association for Computational Linguistics: Human Language Technologies, pages 1534-1544.

Mandar Joshi, Danqi Chen, Yinhan Liu, Daniel S. Weld, Luke Zettlemoyer, and Omer Levy. 2019a. Spanbert: Improving pre-training by representing and predicting spans. CoRR, abs/1907.10529.

Mandar Joshi, Omer Levy, Luke Zettlemoyer, and Daniel Weld. 2019b. BERT for coreference resolution: Baselines and analysis. In Proceedings of the 2019 Conference on Empirical Methods in Natural Language Processing and the 9th International Joint Conference on Natural Language Processing (EMNLP-IJCNLP), pages 5803-5808, Hong Kong, China. Association for Computational Linguistics.

X. J. Kennedy and D. Gioia. 1983. Literature: An introduc-tion to fiction. Poetry, Drama, and writing.

Evgeny Kim and Roman Klinger. 2019. Frowning frodo, wincing leia, and a seriously great friendship: Learning to classify emotional relationships of fictional characters. In Proceedings of the 2019 Conference of the North American Chapter of the Association for Computational Linguistics: Human Language Technologies, NAACL-HLT 2019, Minneapolis, MN, USA, June 2-7, 2019, Volume 1 (Long and Short Papers), pages 647-653. Association for Computational Linguistics.

Vinodh Krishnan and Jacob Eisenstein. 2015. "you're mr. lebowski, I'm the dude": Inducing address term formality in signed social networks. In Proceedings of the 2015 Conference of the North American Chapter of the Association for Computational Linguistics: Human Language Technologies, pages 1616-1626, Denver, Colorado. Association for Computational Linguistics.

Wojciech Kryscinski, Nitish Shirish Keskar, Bryan McCann, Caiming Xiong, and Richard Socher. 2019. Neural text summarization: A critical evaluation. In Proceedings of the 2019 Conference on Empirical Methods in Natural Language Processing and the 9th International Joint Conference on Natural Language Processing (EMNLP-IJCNLP), pages 540551, Hong Kong, China. Association for Computational Linguistics.

Faisal Ladhak, Bryan Li, Yaser Al-Onaizan, and Kathleen McKeown. 2020. Exploring content selection in summarization of novel chapters. In Proceedings of the 58th Annual Meeting of the Association for 
Computational Linguistics, pages 5043-5054, Online. Association for Computational Linguistics.

Zhenzhong Lan, Mingda Chen, Sebastian Goodman, Kevin Gimpel, Piyush Sharma, and Radu Soricut. 2020. Albert: A lite bert for self-supervised learning of language representations. In International Conference on Learning Representations.

J Richard Landis and Gary G Koch. 1977. The measurement of observer agreement for categorical data. biometrics.

Mike Lewis, Yinhan Liu, Naman Goyal, Marjan Ghazvininejad, Abdelrahman Mohamed, Omer Levy, Ves Stoyanov, and Luke Zettlemoyer. 2019. Bart: Denoising sequence-to-sequence pre-training for natural language generation, translation, and comprehension. arXiv.

Chin-Yew Lin. 2004. ROUGE: A package for automatic evaluation of summaries. In Text Summarization Branches Out, pages 74-81, Barcelona, Spain. Association for Computational Linguistics.

Yinhan Liu, Myle Ott, Naman Goyal, Jingfei Du, Mandar Joshi, Danqi Chen, Omer Levy, Mike Lewis, Luke Zettlemoyer, and Veselin Stoyanov. 2019. Roberta: A robustly optimized BERT pretraining approach. CoRR, abs/1907.11692.

Mehdi Manshadi, Reid Swanson, and Andrew S. Gordon. 2008. Learning a probabilistic model of event sequences from internet weblog stories. In Proceedings of the Twenty-First International Florida Artificial Intelligence Research Society Conference, May 15-17, 2008, Coconut Grove, Florida, USA, pages 159-164. AAAI Press.

Joshua Maynez, Shashi Narayan, Bernd Bohnet, and Ryan McDonald. 2020. On faithfulness and factuality in abstractive summarization. In Proceedings of the 58th Annual Meeting of the Association for Computational Linguistics, pages 1906-1919, Online. Association for Computational Linguistics.

Neil McIntyre and Mirella Lapata. 2010. Plot induction and evolutionary search for story generation. In Proceedings of the 48th Annual Meeting of the Association for Computational Linguistics, pages 15621572, Uppsala, Sweden. Association for Computational Linguistics.

Rada Mihalcea and Hakan Ceylan. 2007. Explorations in automatic book summarization. In Proceedings of the 2007 Joint Conference on Empirical Methods in Natural Language Processing and Computational Natural Language Learning (EMNLPCoNLL), pages 380-389, Prague, Czech Republic. Association for Computational Linguistics.

Rada Mihalcea and Paul Tarau. 2004. TextRank: Bringing order into text. In Proceedings of the 2004 Conference on Empirical Methods in Natural Language Processing, pages 404-411, Barcelona, Spain. Association for Computational Linguistics.
Jekaterina Novikova, Ondřej Dušek, Amanda Cercas Curry, and Verena Rieser. 2017. Why we need new evaluation metrics for NLG. In Proceedings of the 2017 Conference on Empirical Methods in Natural Language Processing, pages 2241-2252, Copenhagen, Denmark. Association for Computational Linguistics.

Kishore Papineni, Salim Roukos, Todd Ward, and WeiJing Zhu. 2002. Bleu: a method for automatic evaluation of machine translation. In Proceedings of the 40th Annual Meeting of the Association for Computational Linguistics, pages 311-318, Philadelphia, Pennsylvania, USA. Association for Computational Linguistics.

Dragomir R. Radev. 2001. Experiments in single and multidocument summarization using mead. In In First Document Understanding Conference.

Alec Radford, Jeff Wu, Rewon Child, David Luan, Dario Amodei, and Ilya Sutskever. 2019. Language models are unsupervised multitask learners. OpenAI Blog, 1:8.

Colin Raffel, Noam Shazeer, Adam Roberts, Katherine Lee, Sharan Narang, Michael Matena, Yanqi Zhou, Wei Li, and Peter J. Liu. 2020a. Exploring the limits of transfer learning with a unified text-totext transformer. Journal of Machine Learning Research, 21(140):1-67.

Colin Raffel, Noam Shazeer, Adam Roberts, Katherine Lee, Sharan Narang, Michael Matena, Yanqi Zhou, Wei Li, and Peter J. Liu. 2020b. Exploring the limits of transfer learning with a unified text-totext transformer. Journal of Machine Learning Research, 21(140):1-67.

Michaela Regneri, Alexander Koller, and Manfred Pinkal. 2010. Learning script knowledge with web experiments. In Proceedings of the 48th Annual Meeting of the Association for Computational Linguistics, pages 979-988, Uppsala, Sweden. Association for Computational Linguistics.

Aurko Roy, Mohammad Saffar, Ashish Vaswani, and David Grangier. 2021. Efficient content-based sparse attention with routing transformers. Trans. Assoc. Comput. Linguistics, 9:53-68.

R. Schank and R. Abelson. 1977. Scripts, plans, goals and understanding: An inquiry into human knowledge structures. Lawrence Erlbaum Associates, Hillsdale, NJ.

Shashank Srivastava, Snigdha Chaturvedi, and Tom M. Mitchell. 2016. Inferring interpersonal relations in narrative summaries. In Proceedings of the Thirtieth AAAI Conference on Artificial Intelligence, pages 2807-2813.

Hardik Vala, David Jurgens, Andrew Piper, and Derek Ruths. 2015. Mr. bennet, his coachman, and the archbishop walk into a bar but only one of them gets recognized: On the difficulty of detecting characters 
in literary texts. In Proceedings of the 2015 Conference on Empirical Methods in Natural Language Processing, pages 769-774.

Ashish Vaswani, Noam Shazeer, Niki Parmar, Jakob Uszkoreit, Llion Jones, Aidan N Gomez, Ł ukasz Kaiser, and Illia Polosukhin. 2017. Attention is all you need. In Advances in Neural Information Processing Systems, volume 30. Curran Associates, Inc.

Xin Wang, Wenhu Chen, Yuan-Fang Wang, and William Yang Wang. 2018. No metrics are perfect: Adversarial reward learning for visual storytelling. In Proceedings of the 56th Annual Meeting of the Association for Computational Linguistics (Volume 1: Long Papers), pages 899-909, Melbourne, Australia. Association for Computational Linguistics.

Thomas Wolf, Lysandre Debut, Victor Sanh, Julien Chaumond, Clement Delangue, Anthony Moi, Pierric Cistac, Tim Rault, R'emi Louf, Morgan Funtowicz, and Jamie Brew. 2019. Huggingface's transformers: State-of-the-art natural language processing. arXiv.

Tianyi Zhang, Varsha Kishore, Felix Wu, Kilian Q. Weinberger, and Yoav Artzi. 2020. Bertscore: Evaluating text generation with bert. In International Conference on Learning Representations.

Weiwei Zhang, Jackie Chi Kit Cheung, and Joel Oren. 2019. Generating character descriptions for automatic summarization of fiction. In The Thirty-Third AAAI Conference on Artificial Intelligence, AAAI 2019, pages 7476-7483. AAAI Press.

\section{A Collecting Annotations from Crowd Workers}

To alleviate the limitations of crowd-sourcing and ensure high quality of annotations, we took several steps. First, we conducted a pilot annotation exercise where we (authors) assessed the feasibility of the proposed task on a subset (250 instances) of the data. This pilot annotation helped us set up the task on AMT in a way that would make the task feasible for turkers (e.g. by asking clear concise questions). Second, we designed our setup to avoid annotator fatigue by asking them to read the summary context once and answer questions about all characters in that summary. Third, we ran a few experiments on AMT (before annotating the entire set) where we also included a 'comment" section for turkers to allow them to bring up issues or ambiguities in our setup. We then manually analyzed the results and modified the tasks based on the comments. Finally, after annotating the entire set, we computed inter-annotator agreement as a way to ensure trust in the annotation quality. We found reasonable agreements between annotators as reported in Footnote 10 of the paper. We would also like to mention that we received several comments from the annotators that they found the task very interesting and enjoyable. 


\begin{tabular}{|c|c|c|c|c|c|}
\hline Model & BLEU & ROUGE-1 & ROUGE-2 & ROUGE-L & BERT-F1 \\
\hline \multicolumn{6}{|c|}{ Length Truncated Input } \\
\hline GPT2-L & $0.90 / 0.71 / 0.60 / 0.59$ & 20.33/19.94/18.81/19.39 & $4.34 / 3.60 / 3.36 / 3.32$ & $18.50 / 17.91 / 17.14 / 17.84$ & $76.23 / 80.11 / 76.53 / 75.81$ \\
\hline Longformer & $1.86 / 1.06 / 0.92 / 0.70$ & $24.16 / 21.78 / 20.55 / 20.20$ & $6.80 / 4.62 / 4.33 / 3.98$ & $22.05 / 19.65 / 18.71 / 18.26$ & $85.60 / 84.92 / 84.46 / 84.24$ \\
\hline \multicolumn{6}{|c|}{ Coreference Truncated Input } \\
\hline GPT2-L & $0.82 / 0.63 / 0.53 / 0.58$ & $19.80 / 19.24 / 17.96 / 18.49$ & $3.86 / 3.24 / 3.06 / 3.04$ & $17.79 / 17.39 / 16.46 / 16.62$ & $76.16 / 79.52 / 78.33 / 80.23$ \\
\hline Longformer & $1.78 / 1.09 / 0.77 / 0.65$ & $23.32 / 22.23 / 20.23 / 19.90$ & $6.04 / 4.80 / 3.96 / 3.57$ & $21.41 / 20.22 / 18.16 / 17.70$ & $85.43 / 85.07 / 84.47 / 84.12$ \\
\hline \multicolumn{6}{|c|}{ Full Length Input } \\
\hline Longformer & $2.15 / 1.31 / 1.04 / 0.65$ & $24.47 / 22.56 / 20.90 / 20.63$ & $6.98 / 5.37 / 4.63 / 3.84$ & $21.91 / 20.40 / 18.85 / 18.48$ & $85.66 / 85.11 / 84.57 / 84.35$ \\
\hline
\end{tabular}

Table 8: Breakdown results on subsets of test set with annotated fact coverage as all/most/some/little.

\begin{tabular}{|c|c|}
\hline Generated Description & Comments \\
\hline 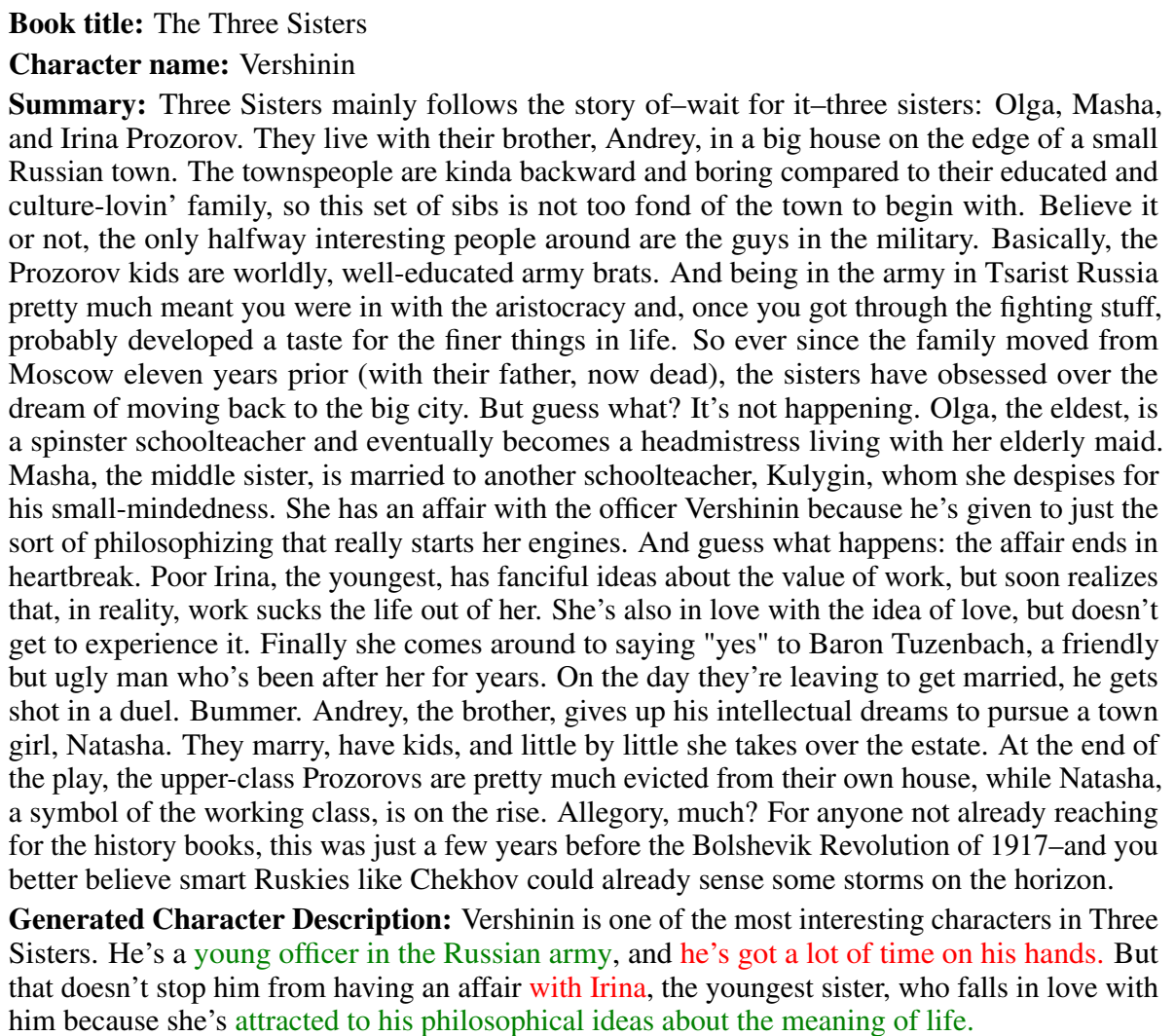 & $\begin{array}{l}\text { According to the sum- } \\
\text { mary "Masha", the mid- } \\
\text { dle sister, is the one who } \\
\text { has an affair with the } \\
\text { officer Vershinin, and } \\
\text { not the youngest sister, } \\
\text { Irina. }\end{array}$ \\
\hline
\end{tabular}

Table 9: Qualitative example 1 for the generated descriptions. Words in red correspond to hallucinated or missing content, words in green correspond to faithful information. 


\begin{tabular}{|c|c|}
\hline Generated Description & Comments \\
\hline 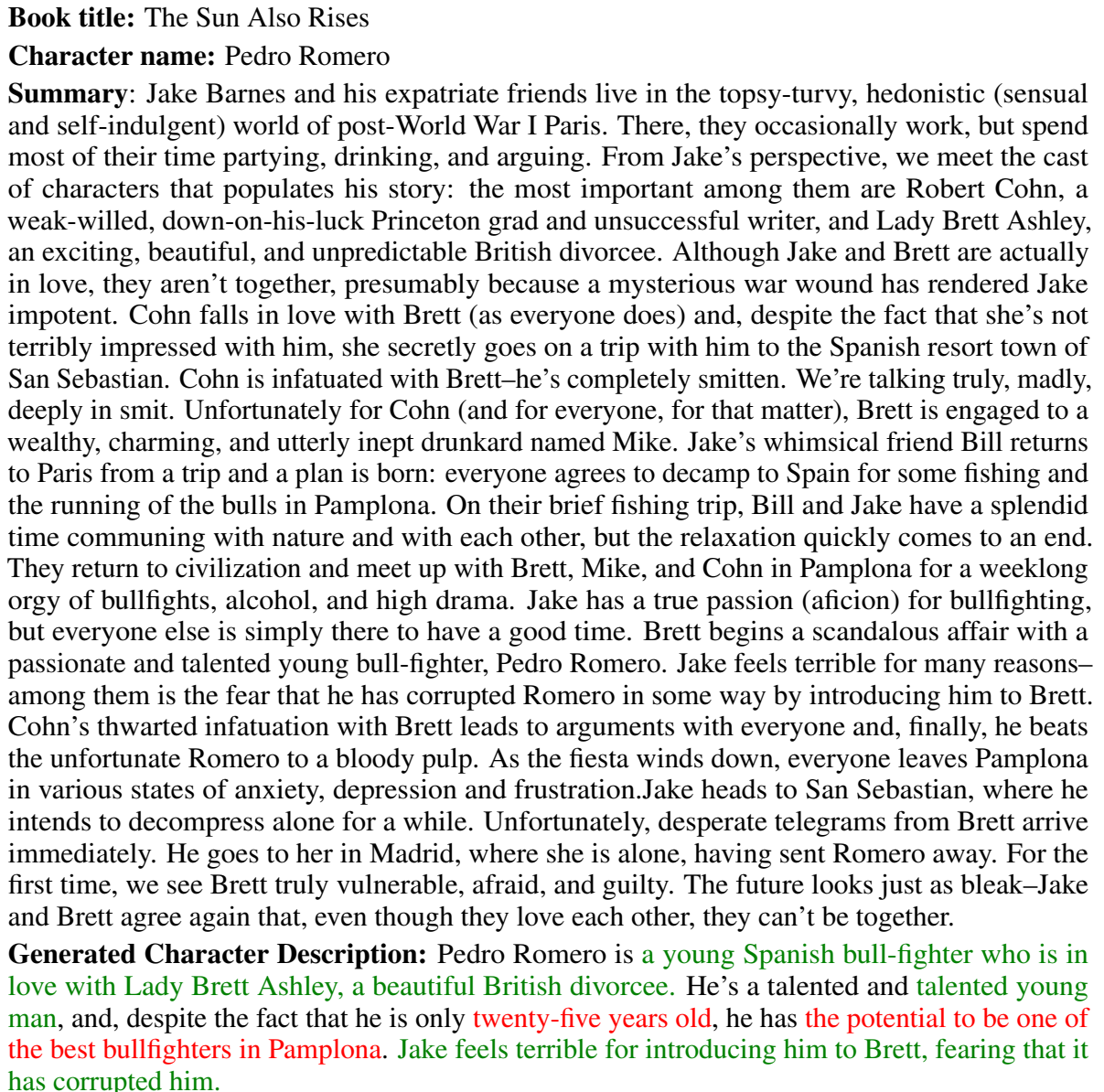 & $\begin{array}{l}\text { The description cap- } \\
\text { tures most of the im- } \\
\text { portant details about the } \\
\text { character. }\end{array}$ \\
\hline
\end{tabular}

Table 10: Qualitative example 2 for the generated descriptions. Words in red correspond to hallucinated or missing content, and words in green correspond to faithful information. 


\begin{tabular}{|c|c|}
\hline Generated Description & Comments \\
\hline 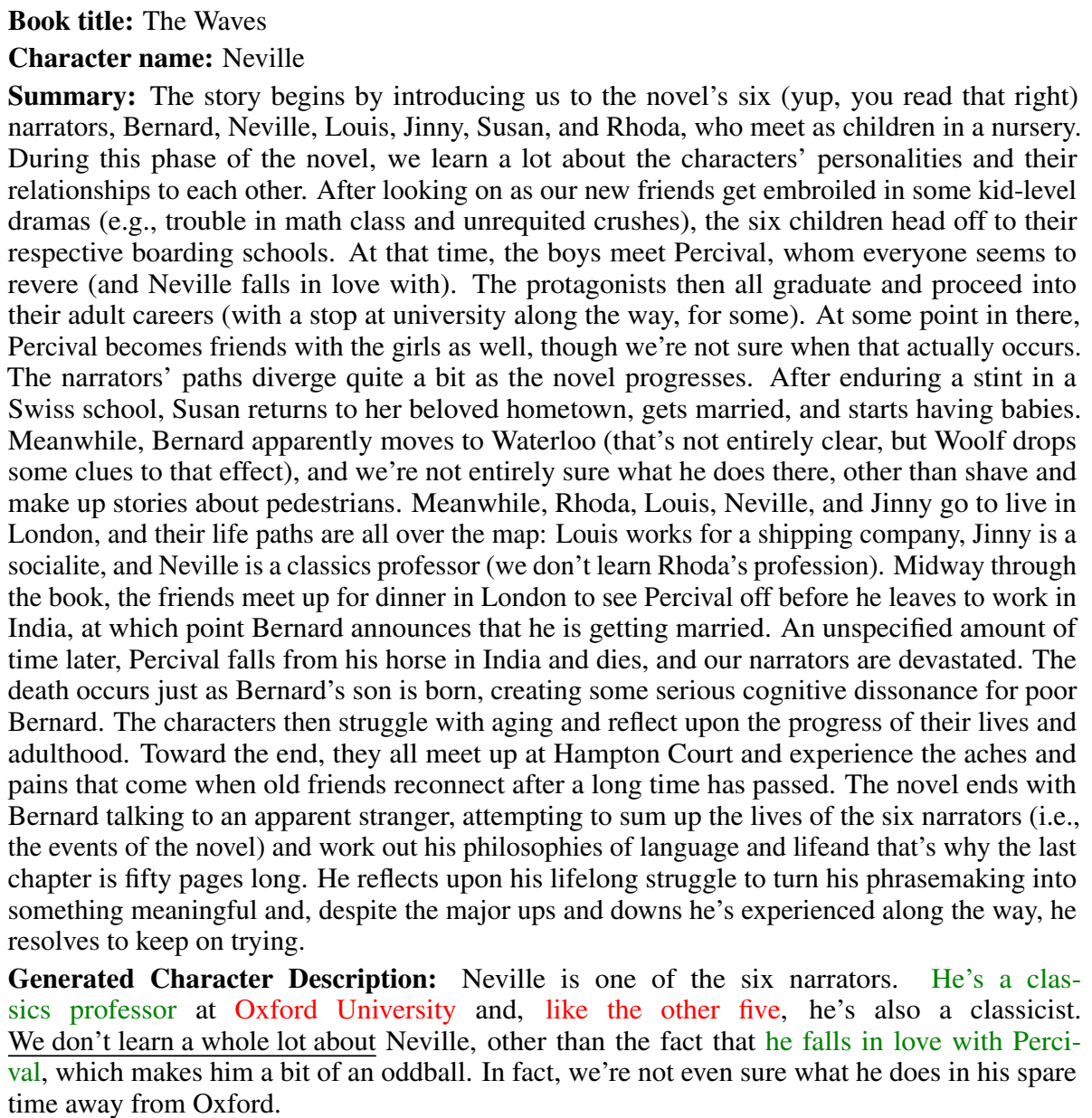 & $\begin{array}{l}\text { The model hallucinated } \\
\text { some details such as be- } \\
\text { ing a professor at Ox- } \\
\text { ford University while } \\
\text { the summary only men- } \\
\text { tions: He lives in Lon- } \\
\text { don and is a classics pro- } \\
\text { fessor. Also, phrases } \\
\text { such as "We don't learn } \\
\text { a whole lot about" ap- } \\
\text { pears quite frequently } \\
\text { in the generated descrip- } \\
\text { tions. }\end{array}$ \\
\hline
\end{tabular}

Table 11: Qualitative example 3 for the generated descriptions. Words in red correspond to hallucinated or missing content, words in green correspond to faithful information, and underline corresponds to generic repetitive content. 


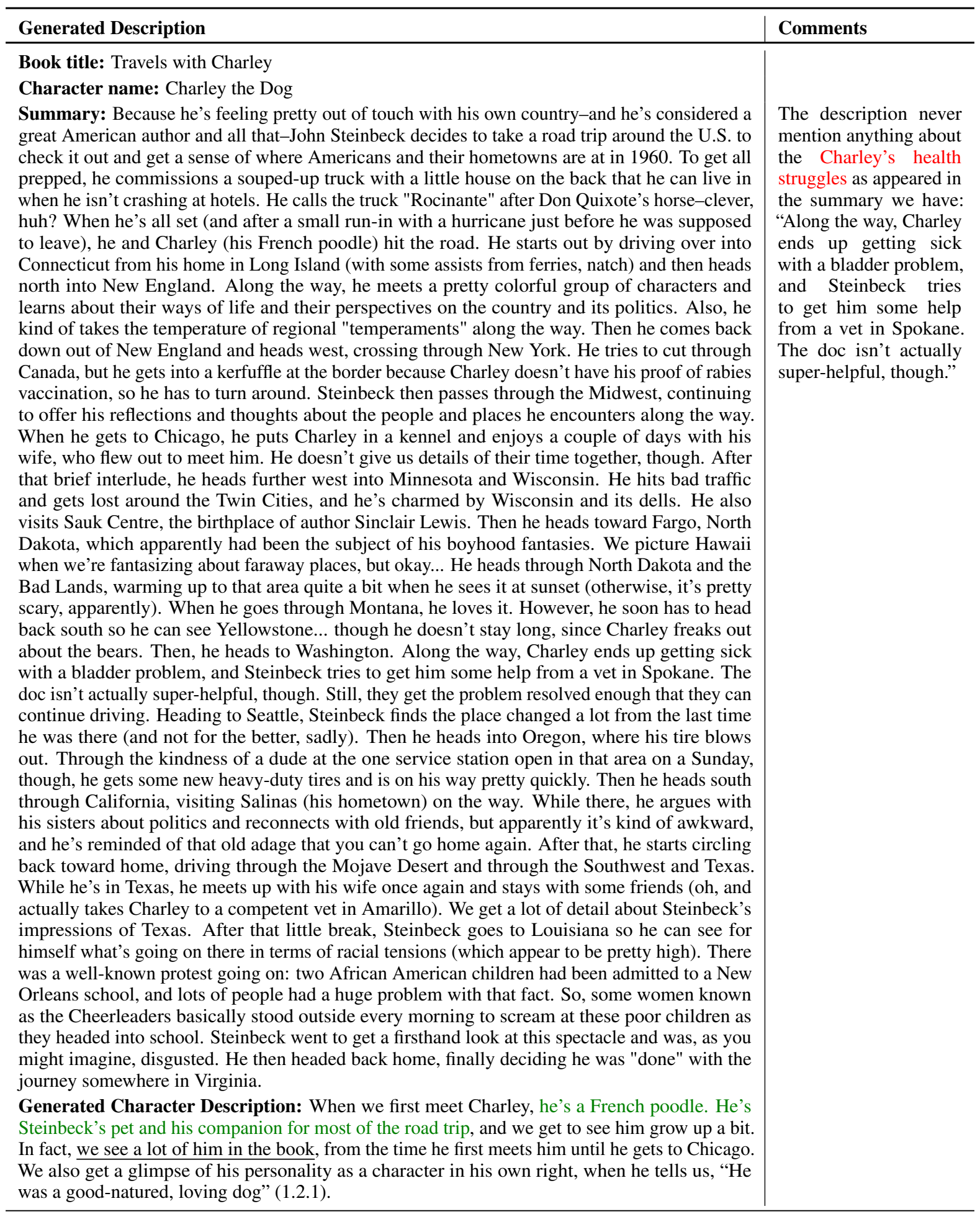

Table 12: Qualitative example 4 for the generated descriptions. words in green correspond to faithful information, and underline corresponds to generic repetitive content. 


\section{Task Description}

In this page, you will be given a book summary along with short descriptions about 4 characters of the book. For each character, you need to do the following 2 tasks:

- Task 1 - In the short character description, all names and mentions of the character (see Notes for more details) are anonymized (replaced with blanks). You need to read the summary and the anonymized character description, then identify which character the description is about by choosing from a list of 5 characters ( 1 extra character choice).

- Task 2 - You will see the full version of the character description this time (character's name and mentions are not anonymized). Read the character description again and evaluate the quality of the character description by answering the following 2 questions:

- How much of the information about the specific character in the corresponding character description is present in the book summary either explicitly or implicitly? Note that "implicit" information can be inferred from the book summary but not directly stated. For example, "Candy adores Mathu because he basically raised her." in character description is implicitly mentioned as "Mathu is virtually her foster father." in the book summary.

Given the book summary, how easy is it to write the character description? If in the previous question you found that some of the information in the character description was not present in the summary, please disregard that while answering this question. In other words, while answering this question please only. consider the information in the character description which is explicitly or implicitly mentioned in the summary.

For each character, Task 2 will appear after you submit the answer for Task 1.

\section{Notes:}

- Make sure you read BOTH the book sumamry and character descriptions carefully, since it will increase the accuracy of the response.

- Character's name and mentions include the following cases:

- Full Name of Character(s)

- First Name of Character(s)

- Nickname/Alias of Character(s), e.g., Captain Americ

Pronouns, e.g., He, She

- Possessive Pronouns, e.g., His, Her

- Noun Phrases that show the relationship of the character(s) with others, e.g., Jeff's Wife

\section{Summary Example}

Three Weeks with My Brother is two stories in one. On the surface, it tells of a trip around the world that Nicholas Sparks takes with his brother Micah. Three Weeks with My Brother begins on the day that Nicholas Sparks receives the flier in the mail and ends with the brothers returning home. In between, Nicholas Sparks recounts the sights, sounds, and spectacles of various countries and continents, taking the reader on the journey with him. But Three Weeks with My Brother is more than just a travelogue. The text is the memoir of a successful author who seemingly is living the American Dream. Yet unbeknownst to most of his readers, he has also lived the American Tragedy. The story follows two brothers and their journey to becoming the best husbands, fathers, sons, brothers, and friends that they can be. Three Weeks with My Brother is a no-holds-barred memoir that shares the good, the bad, and the ugly that made Nicholas Sparks the man he is today.

Task 1 Example

Character Description (anonymized)

Co-author and narrative voice of the memoir. Three Weeks with My Brother is both the story of a trip Micah, as well as the story of his immediate family as takes with brother,

Based on the summary and the character description, answer the following question:

Which character among the following does the description refer to?

A. O Micah Sparks

B. Dana Sparks

C. $\odot$ Nicholas Sparks

D. $\bigcirc$ Bob

E. $\bigcirc$ unable to identify character

\section{Task 2 Example}

Character Description (full version):

Co-author and narrative voice of the memoir. Three Weeks with My Brother is both the story of a trip he takes with his brother, Micah, as well as the story of his immediate family as he grows up, marries, and has children of his own.

Based on the summary and the character description, answer the following question:

How much of the information about the specific character in the corresponding character description is present in the summary (either explicitly or implicitly)?

A. $\bigcirc$ almost all information

B. $\bigcirc$ most of the information

C. $\bigcirc$ some of the information

D. $\bigcirc$ little or none

E. $\bigcirc$ character does not appear in the summary at al

Explanation: The correct answer is "almost all information" since in the given character description, it is mentioned "the story of his immediate family as he grows up, marries, and has children of his own" and same information is implicitly present in the book summary when it says: "The story follows two brothers and their journey to becoming the best husbands, fathers, sons, brothers, and etc.

Given the summary, how easy is it to write the character description? If in the previous question you found that some of the information in the character description was not present in the summary, please disregard that while answering this question. In other words, please only consider the information in the character description which is explicitly or implicitly mentioned in the summary.
Too Difficul
Somewhat
at Difficult
Medium
Somewhat Easy Too Easy

Figure 6: An illustration of human assessment on AMT. 


\section{Task Description}

In this page, you will be given a book summary along with short descriptions about 4 characters of the book. For each character, you need to answer 6 questions that help us to measure how good these descriptions are. The first 4 questions ask you to measure the following 4 quality metrics on a scale of 作

1. Grammatical Correctness (Q1) - A grammatical character description will mostly follow correct English grammar

2. Logical Correctness (Q2) - A logically coherent character description might be grammatically incorrect but will be able to convey the intended

3. Faithfulness (Q3) - A faithful character description will not mention facts, which are irrelevant to the character and/or not stated in the summary

4. Coverage of Key Facts (Q4a) - Whether the character description captures all the key facts about the character in the summary

Question $4 \mathrm{~b}$ asks you to provide what details/facts mentioned in the summary are missing from the descriptions. Please select all that apply.

Question 5 asks you to measure the overall quality of the descriptions by considering all 4 quality metrics describe above on a scale of $\mathbf{1}$ to $\mathbf{5}$ with $\mathbf{1}$ being the lowest quality and 5 being the highest quality.

Notes:

- Make sure you read BOTH the sumamry and descriptions carefully, since it will increase the accuracy of the response.

Example

Summary:

Three Weeks with My Brother is two stories in one. On the surface, it tells of a trip around the world that Nicholas Sparks takes with his brother Micah. Three Weeks with My Brother begins on the day that Nicholas Sparks receives the flier in the mail and ends with the brothers returning home. In between, Nicholas Sparks recounts the sights, sounds, and spectacles of various countries and continents, taking the reader on the journey with him. But Three Weeks with My Brother is more than just a travelogue. The text is the memoir of a successful author who seemingly is living the American Dream. Yet unbeknownst to most of his readers, he has also lived the American Tragedy. The story follows two brothers and their journey to becoming the best husbands, fathers, sons, brothers, and friends that they can be. Three Weeks with My Brother is a no-holds-barred memoir that shares the good, the bad, and the ugly that made Nicholas Sparks the man he is today.

Description :

Nicholas Sparks

Co-author and narrative voice of the memoir. Three Weeks with My Brother is both the story of a trip he takes with his brother, Micah, as well as the story of his immediate family as he grows up, marries, and has children of his own.

Based on the summary and the description, answer the following question:

Q1: Is the character description grammatical?

Please answer it on a scale of 1 to 5 with 1 being "not grammatical at all" and 5 being "very grammatical". A grammatical character description will mostly follow correct English grammar. Note that text can be grammatically correct but nonsensical. For example, "Where did the spoon take off?" should be rated as 5 for this question because it is grammatically correct.

$$
\begin{array}{lllll}
\bigcirc & \bigcirc & \bigcirc & \bigcirc & \bigcirc \\
1 & 2 & 3 & 4 & 5
\end{array}
$$

Q2: Is the character description logically meaningful and coherent?

Please answer it on a scale of 1 to 5 with 1 being "not logically meaningful or coherent at all" and 5 being "very logically meaningful an coherent". A logically coherent character description might be grammatically incorrect but will be able to convey the intended meaning as whole and will make sense. For example, "John and Mary goes to the market." should be rated as 5 for this question because it conveys the intended meaning.

$$
\begin{array}{lllll}
\bigcirc & \bigcirc & \bigcirc & \bigcirc & \bigcirc \\
1 & 2 & 3 & 4 & 5
\end{array}
$$

Q3: Is the character description faithful to the given summary?

Please answer it on a scale of 1 to 5 with 1 being "not faithful at all" and 5 being "very faithful". A faithful character description will not mention facts, which are irrelevant to the character and/or not stated in the summary.

$$
\begin{array}{lllll}
0 & 0 & 0 & 0 & 0 \\
1 & 2 & 3 & 4 & 5
\end{array}
$$

Q4a: Does the character description capture all the key facts about this character in the summary? Please answer it on a scale of 1 to 5 with 1 being "none of the key facts" and 5 being "all of the key facts". These could include (but not restricted to) main events that the character is involved in, the characterâ $\epsilon^{T M} \mathrm{~s}$ role in the narrative, and his/her relationship with other key characters.

$$
\begin{array}{lllll}
\bigcirc & \bigcirc & \bigcirc & \bigcirc & \bigcirc \\
1 & 2 & 3 & 4 & 5
\end{array}
$$

Q4b: What details about the character does the above character description miss or describe incorrectly? (Select all that apply) Note that the description is supposed to describe only the important details and not necessarily all of them.

The character description misses/misrepresents some main event(s) that the character is involved in

The character's role in the narrative (e.g., protagonist, antagonist, etc.) is important but is not included or misrepresented in the character description

The character's relationship with other characters in the narrative is important (e.g., the protagonist's wife) but is not included or misrepresented in the character description

The character's personal characteristics (e.g. age, ethnicity, size, personality type, etc.) are important for the narrative but are not included or misrepresented in the character description

The character's motivation, desires, needs, and behavior are important but are not included or misrepresented in the character description

None of the above. The character description captures most of the important details about the character

Other key points that are missing or misrepresented but not listed above

Q5: Considering all four criteria listed above (question 1 - 4) how would you rate the overall quality of this character's description?

Please answer it on a scale of 1 to 5 with 1 being "not good at all" and 5 being "very good".

$$
\begin{array}{lllll}
\bigcirc & 0 & 0 & \circ & \circ \\
1 & 2 & 3 & 4 & 5
\end{array}
$$

Figure 7: An illustration of human evaluation for generated character description. 